\title{
Mode locking and spatiotemporal chaos in periodically driven Gunn diodes
}

\author{
Mosekilde, Erik; Feldberg, Rasmus; Knudsen, Carsten; Hindsholm, Morten
}

Published in:

Physical Review B

Link to article, DOI:

10.1103/PhysRevB.41.2298

Publication date:

1990

Document Version

Publisher's PDF, also known as Version of record

Link back to DTU Orbit

Citation (APA):

Mosekilde, E., Feldberg, R., Knudsen, C., \& Hindsholm, M. (1990). Mode locking and spatiotemporal chaos in periodically driven Gunn diodes. Physical Review B, 41(4), 2298-2306.

https://doi.org/10.1103/PhysRevB.41.2298

\section{General rights}

Copyright and moral rights for the publications made accessible in the public portal are retained by the authors and/or other copyright owners and it is a condition of accessing publications that users recognise and abide by the legal requirements associated with these rights.

- Users may download and print one copy of any publication from the public portal for the purpose of private study or research.

- You may not further distribute the material or use it for any profit-making activity or commercial gain

- You may freely distribute the URL identifying the publication in the public portal 


\title{
Mode locking and spatiotemporal chaos in periodically driven Gunn diodes
}

\author{
Erik Mosekilde, Rasmus Feldberg, Carsten Knudsen, and Morten Hindsholm \\ Physics Laboratory III, The Technical University of Denmark, DK-2800 Lyngby, Denmark
}

(Received 10 July 1989)

\begin{abstract}
Numerical simulation is applied to study the highly nonlinear-dynamic phenomena that can arise in Gunn diodes by interaction between the internally generated domain mode and an external microwave signal. By adjusting the time of domain formation and the speed of propagation, the internal oscillation entrains with the external signal. This produces a devil's staircase of frequencylocked solutions. At higher microwave amplitudes, period doubling and other forms of modeconverting bifurcations can be seen. In this interval the diode also exhibits spatiotemporal chaos. At still higher microwave amplitudes, transitions to delayed, quenched, and limited space-charge accumulation modes take place.
\end{abstract}

\section{INTRODUCTION}

Instabilities in dissipative systems can give rise to a wealth of complex nonlinear-dynamic phenomena, including (i) cascades of period-doubling bifurcations, (ii) entrainment between an internally generated oscillation and an external signal, and (iii) various types of deterministic chaos. The study of these phenomena has attracted a rapidly growing interest during the past decade, and examples of chaotic behavior have been reported from almost all branches of science..$^{1-5}$ A considerable number of studies have been concerned with nonlineardynamic phenomena in electronic circuits, ${ }^{6-8}$ including circuits involving semiconductor devices such as tunneling $^{9}$ and varactor diodes. ${ }^{10}$ In these studies, the active element has typically been characterized by means of a time-independent current-voltage characteristic. In no case, it appears, has the extended nature of the device or its complex internal dynamics been taken into account.

It is well known that $n$-type GaAs and a number of other compound semiconductors can exhibit selfsustained current oscillations in the microwave range when the applied drift field exceeds a characteristic threshold value. ${ }^{11}$ Because of inefficient energy relaxation, the electron gas heats up to temperatures well above that of the crystal lattice, and a transfer of carriers from the high-mobility conduction-band minimum to a set of low-mobility satellite valleys takes place. If this transition is fast enough, a bulk negative differential conductivity may arise. The spatially homogeneous electron distribution then becomes unstable, and propagating highfield domains are formed. ${ }^{12}$ In the external circuit, the formation and propagation of these domains give rise to current oscillations with a typical frequency of $7-9 \mathrm{GHz}$ for a $12-\mu \mathrm{m}$ sample.

The Gunn effect has been extensively studied by experimental ${ }^{13}$ and various analytical and numerical techniques. ${ }^{14-16}$ In particular, Copeland ${ }^{17}$ has shown that the presence of a microwave signal of sufficient amplitude and frequency can suppress the formation of domains and produce an alternative mode of operation for the Gunn diode, which is referred to as a limited space-charge accu- mulation (or LSA) mode. This mode allows high dc-to-rf conversion efficiencies and worthwhile microwave output powers to be attained in the $50-120-\mathrm{GHz}$ regime.

Based upon a relatively detailed model for the formation and propagation of subsequent high-field domains, this paper describes the highly nonlinear-dynamic phenomena that can arise in a periodically driven Gunn diode. As the frequency of the applied microwave signal is changed, a devil's staircase of frequency-locked oscillations develops. At higher microwave amplitudes, period doubling and other forms of mode-converting bifurcations can be seen. In this regime, spatiotemporal chaos is also observed. For even higher microwave amplitudes, transitions to delayed, quenched and limited space-charge accumulation modes take place. Several of these modes have been experimentally observed. However, the detailed distribution of modes in parameter space does not appear to have previously been obtained.

\section{THE TWO-VALLEY MODEL OF DOMAIN FORMATION}

The Gunn effect is associated with a transfer of electrons from the high-mobility conduction-band minimum to a set of low-mobility satellite valleys approximately $\Delta=0.35 \mathrm{eV}$ higher in the conduction band. For small applied fields, only the low-lying $\Gamma$ minimum is populated, and the current-voltage relation is approximately Ohmic. As the drift field is increased, the electron gas starts to heat up, and at a threshold field of about 3.5 $\mathrm{kV} / \mathrm{cm}$ (for GaAs), the average electron energy becomes sufficient for a population of the higher-lying satellite valleys to begin. These valleys have an effective density of states that exceeds that of the conduction-band minimum by a factor of the order of $60,{ }^{14}$ and at sufficiently high electric fields, a majority of the electrons will therefore occupy states in the satellite valleys.

Direct experimental evidence for the electron-transfer mechanism was first established by Hutson et al., ${ }^{18}$ who observed a reduction in the threshold field for current oscillations as the valley separation $\Delta$ was reduced under hydrostatic pressure. Detailed calculations by 
McCumber and Chynoweth ${ }^{14}$ and by Kroemer ${ }^{15}$ have subsequently shown that the two-valley model can satisfactorily account both for the observed small-signal instability and for the formation of traveling high-field domains. The purpose of the present paper is to investigate the nonlinear dynamic phenomena that arise when the formation of domains takes place in the presence of an external microwave signal. For this purpose a slightly simplified version of the two-valley model suffices. In particular, to avoid calculation of the temperature of the electron gas, we have applied results for the equilibrium distribution of electrons between the two sets of valleys obtained through detailed Monte Carlo calculations of electron transport properties in GaAs. ${ }^{19}$

Let us consider a one-dimensional model of a GaAs Gunn diode of length $L$. The electric field is applied along the $x$ direction, and we assume that electrons are distributed in the high- and low-mobility states with concentrations $n_{1}(x, t)$ and $n_{2}(x, t)$, respectively. For simplicity, the electrons are assumed to carry a positive charge $e$. The total current density $j(t)$ is considered to consist of drift and diffusion terms from each of the two groups of electrons. In addition, there is a dielectric displacement term that secures conservation of charge. Thus $^{20}$

$j=n_{1} e v_{1}(F)+n_{2} e \mu_{2} F-e D_{1} \frac{\partial n_{1}}{\partial x}-e D_{2} \frac{\partial n_{2}}{\partial x}+\epsilon \frac{\partial F}{\partial t}$,

where $F=F(x, t)$ is the local electric field as determined from Poisson's equation

$$
\frac{\partial F}{\partial x}=\frac{e}{\epsilon}\left(n_{1}+n_{2}-n_{0}\right) .
$$

Here, $\epsilon$ is the static dielectric constant, and $n_{0}$ is the thermal equilibrium electron concentration. In the numerical calculations, we have taken $n_{0}=2 \times 10^{15} / \mathrm{cm}^{3}$, and the relative dielectric constant for GaAs has been inserted as $\epsilon_{r}=12.5$. The assumptions of a constant diffusion constant and a linear relation between velocity and drift field for electrons in the satellite valleys agree well with numerical results. ${ }^{19,21}$ Because of relatively efficient intervalley scattering, the electron distribution in the upper valleys remains almost Maxwellian, with a temperature close to that of the crystal lattice. The values that we have chosen for the mobility $\mu_{2}=320 \mathrm{~cm}^{2} / \mathrm{V} \mathrm{s}$ and diffusion constant $D_{2}=8 \mathrm{~cm}^{2} / \mathrm{s}$ also seem to be in agreement with numerical calculations.

Although common in studies of high-field domain formation, ${ }^{15}$ the assumption of a constant diffusion constant for electrons in the conduction-band minimum is more difficult to justify. Diffusion plays a relatively significant role as a mechanism that stabilizes the spatially homogeneous electron distribution, and thus counteracts domain formation. Without diffusion, the electron density gradients in the Gunn domain could become infinitely large. Diffusion also influences the velocity of the stably propagating domain. ${ }^{16,20}$ However, as long as the magnitude of the diffusion effects is approximately correct, the variation of these effects with field are presumably less significant. In our numerical calculations we have taken
$D_{1}=200 \mathrm{~cm}^{2} / \mathrm{s}$.

Using detailed Monte Carlo techniques, Fawcett et al. ${ }^{19}$ have calculated the stationary electron transport properties of GaAs as a function of the applied field. With these techniques, the motion of a single electron is simulated through a large number of scattering processes, and the time spent by the electron in each little element of momentum space is recorded. After a sufficient number of scattering processes, the distribution of visiting times is taken as being expressive of the distribution of electrons. The scattering processes are simulated by drawing random numbers for the time of flight between collisions, for the actual scattering mechanism, and for the final state in the scattering process, all in accordance with theoretically derived probability distributions for these processes.

In their calculations for $n$-type GaAs, Fawcett et al. ${ }^{19}$ included polar-optical and acoustic-phonon scattering for electrons in the conduction-band minimum, intravalley and equivalent intervalley scattering for electrons in the satellite valleys, as well as nonequivalent scattering of electrons between the two sets of valleys. Nonparabolicity of the conduction-band minimum and wave-vector dependence of the periodic part of the Bloch functions for electrons in this minimum were also included.

Figure 1 reproduces results obtained by Fawcett et al. ${ }^{19}$ for the fraction of electrons that occupy states in the satellite valleys as a function of the drift field. These results apply under stationary and spatially homogeneous conditions without domain formation. The fraction of electrons in the upper valleys is very sensitive to the value of the nonequivalent intravalley deformation potential $\Xi_{i j}$. By comparing with experimental results obtained among others, i.e., Ruch and Kino, ${ }^{22}$ Fawcett et al. conclude that a value of $\Xi_{i j}=1 \times 10^{9} \mathrm{eV} / \mathrm{cm}$ gives the best overall fit to the total velocity-field characteristic. In our calculations we have assumed a simple analytical relation

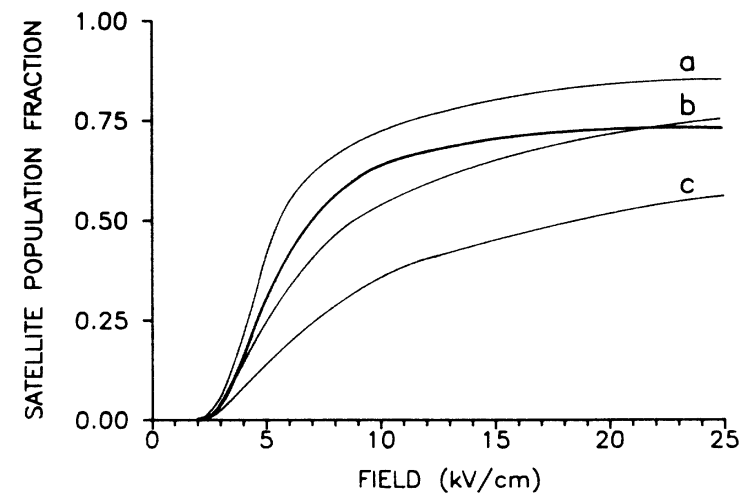

FIG. 1. Fraction of electrons that occupy states in the satellite valleys as a function of the drift field. Fine curves obtained by detailed Monte Carlo calculations (Ref. 19) for different values of the nonequivalent intervalley deformation potential (a) $\Xi_{i j}=2.0 \times 10^{9} \mathrm{eV} / \mathrm{cm}$, (b) $1.0 \times 10^{9} \mathrm{eV} / \mathrm{cm}$, and (c) $0.5 \times 10^{9}$ $\mathrm{eV} / \mathrm{cm}$. These results apply under stationary and spatially homogeneous conditions. The heavy curve shows the simplified analytical approximation applied in the present study. 
for the fraction $n_{20}(F) / n_{0}$ of electrons in the upper valleys under conditions of equilibrium at the local field. This analytical approximation is shown by the heavy curve in Fig. 1.

Similarly, we have assumed a simple analytical relation for the velocity-field characteristic $v_{1}(F)$ of electrons in the conduction-band minimum. This relation has been adjusted such that the low-field mobility is $\mu_{1}=5000 \mathrm{~cm}^{2} / \mathrm{V} \mathrm{s}$, and the saturation drift velocity for fields in excess of $4.0 \mathrm{kV} / \mathrm{cm}$ is $v_{1 s}=2.3 \times 10^{7} \mathrm{~cm} / \mathrm{s}$. This again corresponds to the results of the detailed Monte Carlo calculations ${ }^{19}$ for $\Xi_{i j}=1 \times 10^{9} \mathrm{eV} / \mathrm{cm}$. For higher values of $\Xi_{i j}$, Fawcett $e t$ al. found a negative differential conductivity for electrons in the conduction-band minimum considered alone. With these approximations for $n_{20}(F) / n_{0}$ and $v_{1}(F)$, the resulting velocity-field characteristic will be in good agreement with experimental curves up to the highest-field strengths arising in our calculations.

Under the dynamical conditions considered in the present model, population equilibration between the two sets of valleys is expressed by

$$
\frac{\partial n_{2}}{\partial t}=\frac{n_{20}(F)-n_{2}(x, t)}{\tau},
$$

with a relaxation time $\tau=2$ ps. The rates at which hot electrons scatter from the conduction-band minimum to the higher-lying $L$ and $X$ valleys in GaAs have recently been measured by Ulbrich et $a l^{23}$ They found scattering times of $\tau_{\Gamma L}=0.48 \mathrm{ps}$ (for $0.48 \mathrm{eV}$ electrons) and $\tau_{\Gamma X}=0.16 \mathrm{ps}$ (for $0.58 \mathrm{eV}$ electrons). In calculations of the response of the electron gas to changes in the applied field, Rees ${ }^{21}$ concludes that this response is controlled by the relatively slow relaxation of electrons in the central valley for energies between 0.1 (below which polar optical scattering is strong) and $0.35 \mathrm{eV}$ (above which intervalley scattering is significant). This picture seems to agree with the observation by Kash et al. ${ }^{24}$ that an electron scattered into the central valley relaxes towards the bottom of this valley through a cascade of typically 10-12 optical phonon emission processes, each requiring approximately 165 fs. Also McCumber and Chynoweth ${ }^{14}$ in their description of the dynamics of electron heating arrive at response times of the order of 2 ps.

As boundary conditions we have assumed thermal equilibrium

$$
n_{1}(0, t)=n_{0} \text { and } n_{2}(0, t)=0
$$

at the cathode end, and neutrality

$$
n_{1}(L, t)+n_{2}(L, t)=n_{0}
$$

at the anode end. Here $L=12 \mu \mathrm{m}$ denotes the length of the sample. Our results are not sensitive to the precise form of these conditions, however. To facilitate the formation of domains, we have assumed that there is a slight $(0.1 \%)$ enhancement of the specific resistance in that $1 \%$ of the crystal that is closest to the cathode end. Finally, we have applied the normalization condition

$$
\int_{0}^{L} F(x, t) d x=V_{\mathrm{dc}}[1+A \sin (2 \pi f t)],
$$

where $V_{\mathrm{dc}}$ is the applied dc voltage. $f$ and $A$ denote the frequency and the relative amplitude of the applied microwave signal, respectively. In all calculations we have taken the average dc field to be $V_{\mathrm{dc}} / L=4.0 \mathrm{kV} / \mathrm{cm}$.

Figure 2 illustrates the formation of the first high-field domain as calculated by the above model. Figure 2(a) shows the variation of the electric field $F(x, t)$ along the crystal at different times after a dc-voltage corresponding to an average field strength of $4 \mathrm{kV} / \mathrm{cm}$ has been applied. Figure 2(b) shows the corresponding variation in the total electron concentration $n_{1}(x, t)+n_{2}(x, t)$, and Figs. 2(c) and $2(\mathrm{~d})$ show the variation in the lower and upper valley populations $n_{1}(x, t)$ and $n_{2}(x, t)$, respectively.

Immediately after the voltage is applied, the field and carrier distributions are uniform, the assumed small irregularity close to the cathode end being invisible on the scales of Fig. 2. Within a period of the order of the intervalley relaxation time, $\tau$, a redistribution of carriers between the two sets of valleys takes place. By virtue of the negative differential conductivity created by this redistribution, the crystal hereafter yields to a convective instability: Small random fluctuations in the carrier distribution become unstable and grow to form a traveling dipole layer with carrier depletion in the leading edge and accumulation of carriers in the trailing edge.

As the carrier-density-electric-field fluctuations become sufficiently large, nonlinear restraining mechanisms set in. The increasing voltage drop across the domain forces the field outside the domain down into the Ohmic region. This sets a stop to the formation of competing domains. At the same time, the domain itself stops to grow, and it hereafter propagates stably towards the anode end of the crystal. For longer samples, the depletion of the electron gas in the leading edge of the domain may be almost complete. A more detailed picture can be obtained by following the variation of $n_{1}(x, t)$ and $n_{2}(x, t)$ separately, as shown in Figs. 2(c) and 2(d). Through the agreement between the numerical results and our physical intuition, these figures serve as a check on the model.

Simulating the model over a somewhat longer period of time gives the results presented in Fig. 3. Here, the upper panel shows the stationary current oscillations that persist in the crystal after the initial transient has died out. The lower panel shows the formation and propagation of subsequent high-field domains. In this panel, the vertical axis represents the position along the crystal with the cathode end at the bottom. The time axis is common to both panels. At a given time, the contour curves in the lower panel demarcate that part of the crystal in which the field is more than $10 \%$ higher than in the rest of the crystal. The current density is seen to rise to its Ohmic value of about $65 \mathrm{~A} / \mathrm{mm}^{2}$ each time a domain disappears at the anode contact. The current remains high during the initial phases of the formation of a new domain. However, as the domain reaches macroscopic significance, the current density decays to a saturated value of about $40 \mathrm{~A} / \mathrm{mm}^{2}$, and the current remains saturated during the period of stable domain propagation. This again agrees with our physical intuition as established through earlier work in the field. The undisturbed 

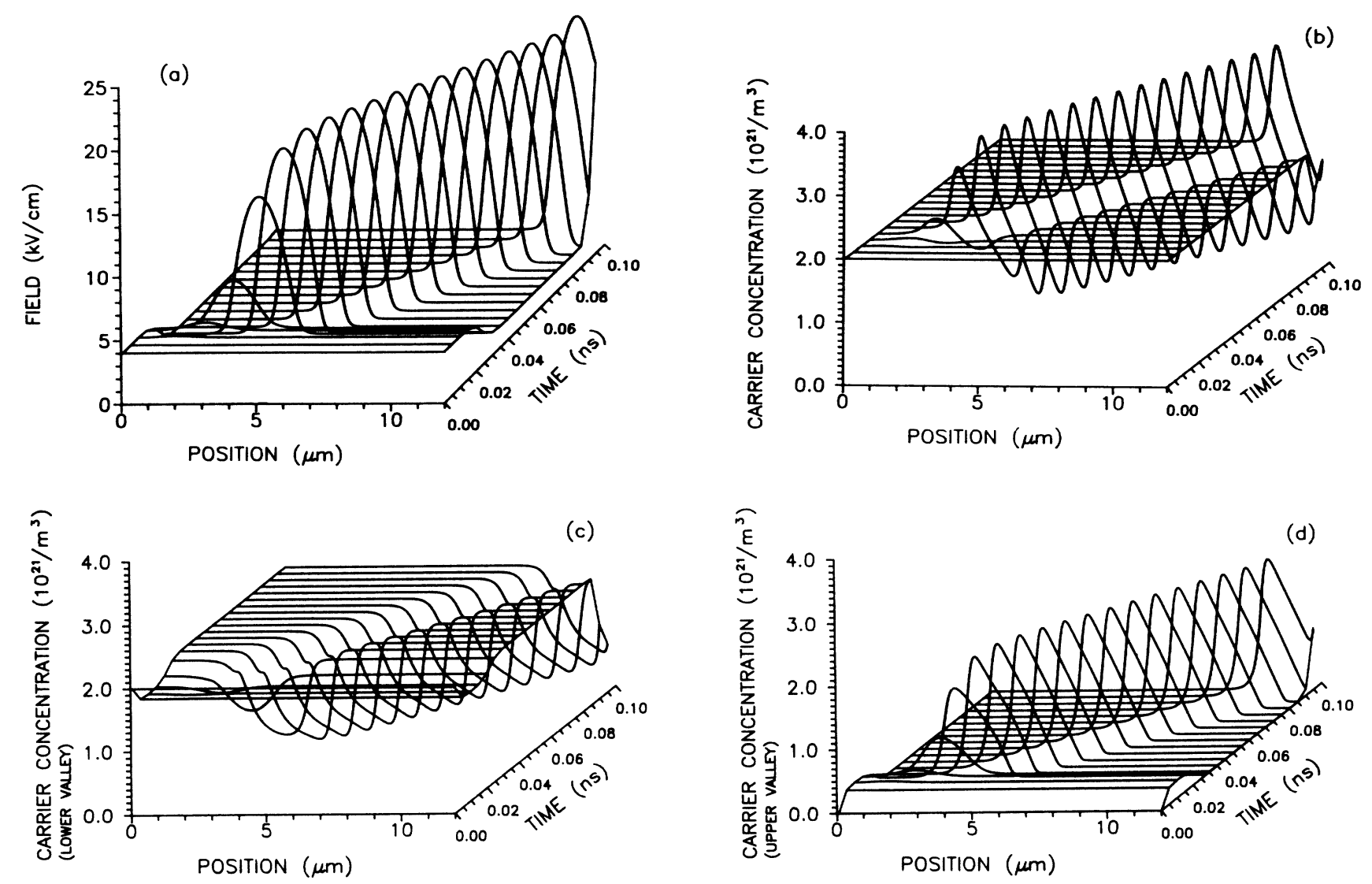

FIG. 2. Formation of the first high-field domain after application of a drift field of $4 \mathrm{kV} / \mathrm{cm}$. (a) shows the temporal and spatial variation of the local electric field $F(x, t)$, (b) shows the corresponding variation of the total carrier density $n_{1}(x, t)+n_{2}(x, t)$, and (c) and (d) show the variations of the

period of oscillation is $0.12 \mathrm{~ns}$, corresponding to a frequency of $f_{\mathrm{Gunn}}=8.7 \mathrm{GHz}$.

\section{MODE LOCKING AND SPATIO-TEMPORAL CHAOS}

By simulating the formation of domains in the presence of a microwave field, we have observed a large variety of different modes of behavior, depending upon the amplitude and frequency of the microwave signal. As an example, Fig. 4 presents the response of the Gunn diode to the application of a microwave signal of frequency $f=12.5 \mathrm{GHz}$ and relative amplitude $A=0.20$. For comparison with Fig. 3, Fig. 4(a) shows the temporal variation of the current density together with contour curves, illustrating the formation and propagation of subsequent high-field domains. Figure $4(\mathrm{~b})$ shows the stationary phase-space trajectory obtained by plotting simultaneous values of the current density and the average electric field

$$
F_{\mathrm{av}}=\left(V_{\mathrm{dc}} / L\right)[1+A \sin (2 \pi f t)]
$$

over many oscillation periods.

The microwave signal is applied at time $t=0.4 \mathrm{~ns}$. After a transient of approximately 0.3-ns's duration, the

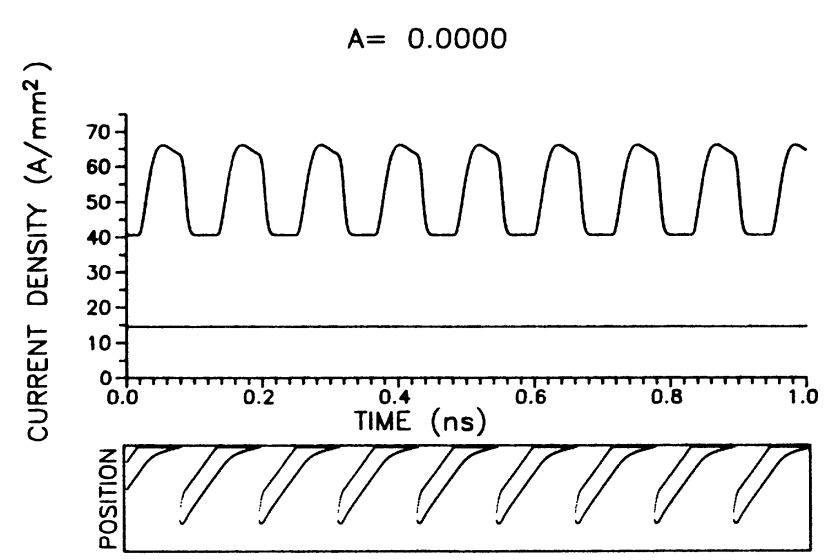

FIG. 3. Stationary current oscillation (upper panel) in a GaAs Gunn diode. The lower panel shows the formation and propagation of subsequent high-field domains.

lower and upper valley populations $n_{1}(x, t)$ and $n_{2}(x, t)$, respectively. For longer crystals, the depletion of carriers at the leading edge of the domain may be nearly complete.

domain mode entrains into a 2:1 frequency-locked solution with the applied microwave field. This occurs by adjusting both the time of domain formation and the speed of propagation. The position in the crystal at which the domain can first be detected is also changed. From the 


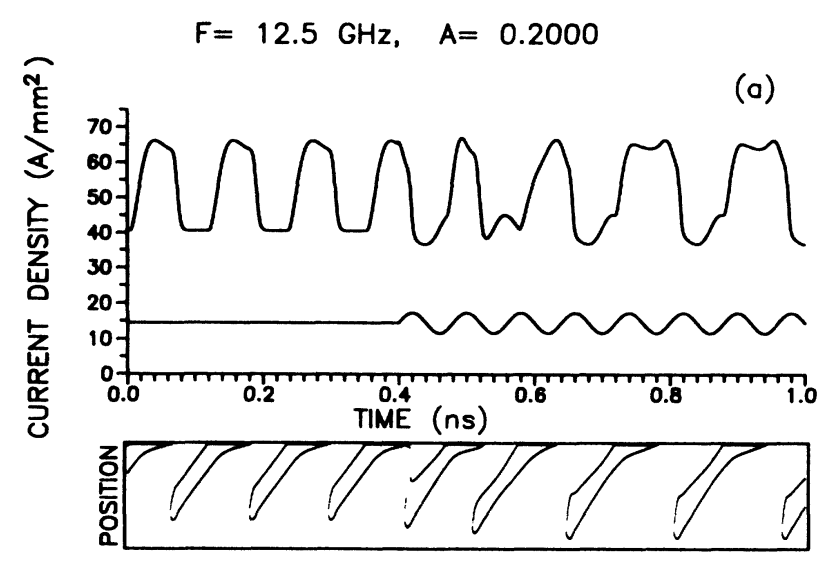

$F=12.5 \mathrm{GHz}, \quad A=0.2000$

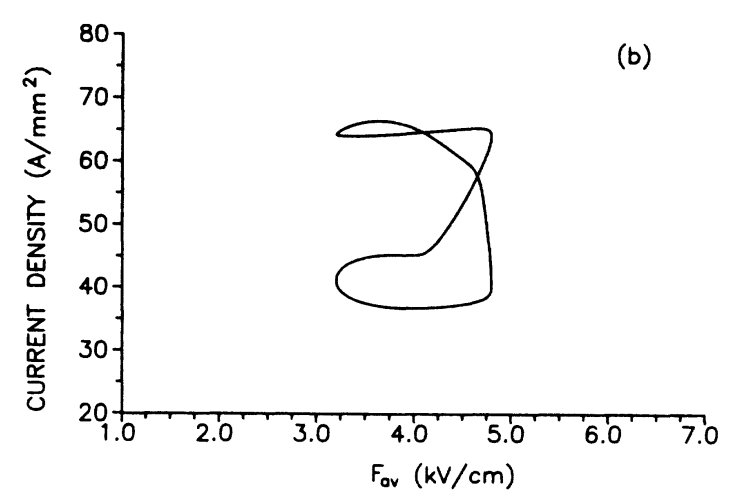

FIG. 4. Onset of a 2:1 frequency-locked solution upon application of a microwave signal of frequency $f=12.5 \mathrm{GHz}$ and relative amplitude $A=0.20$. (b) Shows a phase plot for the stationary 2:1 solution. By comparison with the undisturbed domain mode of Fig. 3, it is observed how the domains adjust their time of formation and their speed of propagation so as to entrain with the microwave signal.

phase plot it is observed how the current drops during the formation of a domain, remains low during a negative swing of the microwave signal, and then rises again as the domain leaves the crystal. Awaiting that, the average field $F_{\text {av }}$ becomes sufficiently high for a new domain to be formed, the current density remains high during a second negative swing of the microwave signal. The Gunn mode thus primarily adjusts to the microwave signal by delaying the formation of a new domain. At the same time, however, the speed of domain propagation is slightly reduced, and all together the reduction in the frequency of the domain mode amounts to approximately $27 \%$.

As the frequency of the microwave signal is increased, the 2:1 frequency-locked solution is maintained up to about $18.4 \mathrm{GHz}$. At this frequency, the period during which the microwave field remains positive after the exit of a domain becomes too short for a new domain to be formed, and the oscillation becomes quasiperiodic. This is illustrated by the series of phase plots in Fig. 5 . $f=12.00 \mathrm{GHz} \quad f=13.00 \mathrm{GHz} \quad f=14.00 \mathrm{GHz} \quad f=15.00 \mathrm{GHz}$

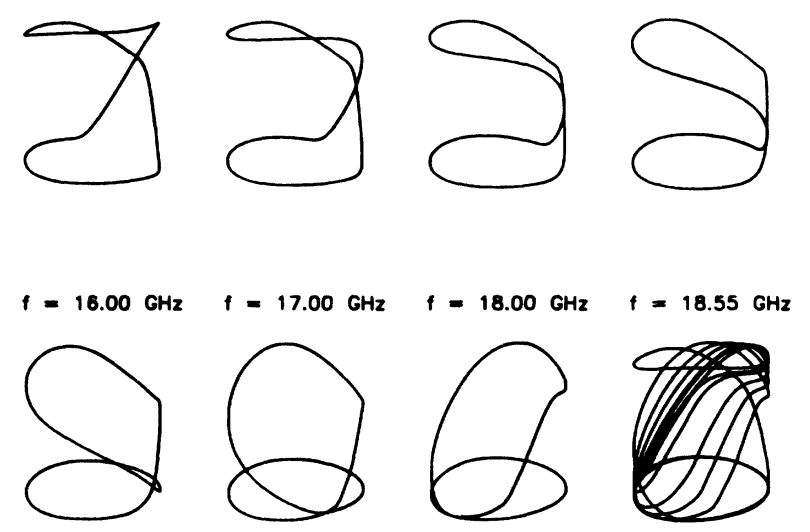

FIG. 5. Series of phase plots obtained by increasing the microwave frequency from 12 to $18.55 \mathrm{GHz}$. The Gunn mode maintains a 2:1 frequency-locked solution up to microwave frequencies of approximately $18.4 \mathrm{GHz}$. At $f=18.55 \mathrm{GHz}$, a quasiperiodic solution (or a periodic solution with a period longer than $5 \mathrm{~ns}$ ) is observed.

For higher microwave frequencies, intervals exist in which the domain mode entrains into $3: 1,4: 1,5: 1$, etc., frequency-locked solutions. As an example, Fig. 6 shows the 6:1 frequency-locked solution that exists for $f=50.0$ $\mathrm{GHz}$ and $A=0.20$. With this solution, the microwave signal performs three oscillations during the period of domain formation, and three oscillations during the period of domain propagation. In the lower panel of Fig. 6(a) we observe how the width of the domain and its speed of propagation are modulated by the microwave signal. It is also interesting to observe how a new domain is nearly formed during the second positive swing of the microwave signal in the high-current state, and then almost quenched to finally reappear and stabilize during the next positive swing of this signal.

In between the main tongues, more complex frequency-locked solutions are observed. As an example Fig. 7 shows the 8:7 frequency-locked solution obtained for $A=0.40$ and $f=10.0 \mathrm{GHz}$. Altogether, the interaction between the internally generated domain mode and the external microwave signal produce the Arnol'd tongue diagram, characteristic of periodically perturbed nonlinear oscillators. ${ }^{25,26}$ For fixed microwave amplitude, the interaction gives rise to a devil's staircase of frequency-locked solutions, interspersed with quasiperiodic behavior. ${ }^{27}$

With increasing microwave amplitudes, the tongues broaden. Since it is easier to delay domain formation than to speed up this process, the broadening is most significant on the low-frequency side of the tongues. In the region where the tongues start to overlap, various forms of period-doubling and mode-converting bifurcations take place. A more detailed investigation of the region between the $3: 1$ and $4: 1$ tongues has thus revealed the existence of 13:4, 10:3, 14:4, 7:2, 11:3, and 8:2 frequency-locked solutions. In this region, we also find 


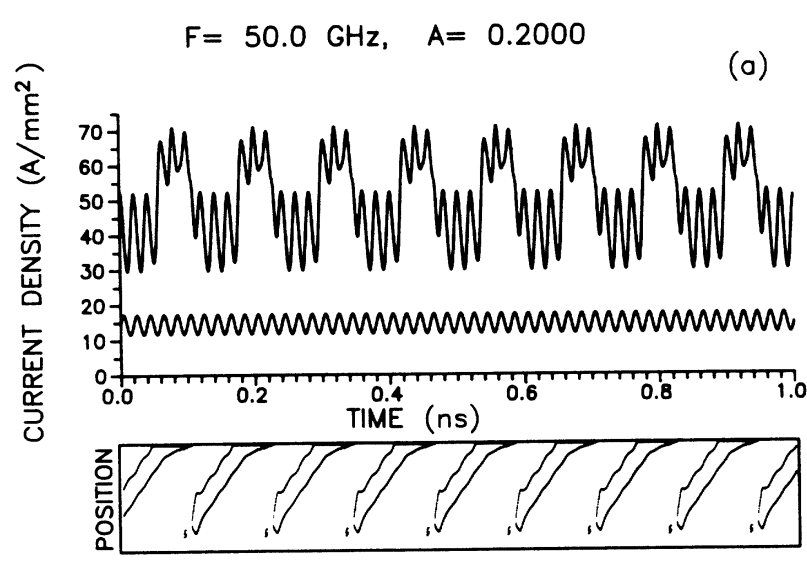

$F=50.0 \mathrm{GHz}, \quad A=0.2000$

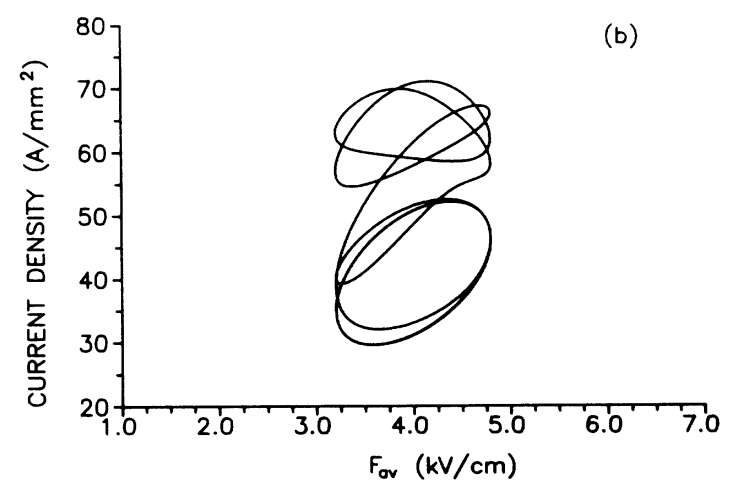

FIG. 6. Stationary 6:1 frequency-locked solution existing for $f=50.5 \mathrm{GHz}$ and $A=0.20$. (a) Temporal variation of current density, applied field, and internal field distribution; (b) corresponding phase-space trajectory.

chaotic solutions. Figure 8(a), for instance, shows a phase plot for the attractor existing for $A=0.56$ and $f=30.0 \mathrm{GHz}$. Figure 8 (b) shows the corresponding temporal variation of the current density and electric-field distribution, and Fig. 8(c) shows a stroboscopic map obtained by plotting simultaneous values of the current density and the rate of change for this variable each time the microwave signal has completed a full oscillation. These figures illustrate the irregular temporal behavior and the characteristic folding of the attractor.

To illustrate the spatially chaotic nature of the solution, Fig. 9(a) shows a stroboscopic map obtained by plotting simultaneous values of the electric field in two points of the crystal situated $x_{1}=L / 2$ and $x_{2}=3 L / 4$ from the cathode contact, respectively. The insert illustrates the layered structure of the chaotic attractor in more detail. Figure 9(b) shows a similar stroboscopic map obtained by plotting simultaneous values of the electric field in two points of the crystal situated $x_{1}=3 L / 8$ and $x_{2}=5 L / 8$ from the cathode contact. In this figure we have illustrated how three neighboring points $a_{1}, a_{2}$, and $a_{3}$ move on the attractor under subsequent itterations. After nine periods of the external microwave signal, $a_{1}$ has been

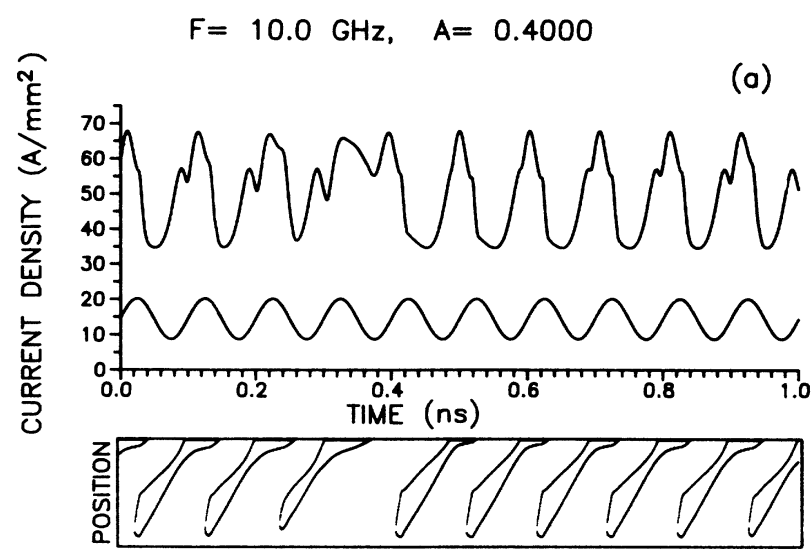

$F=10.0 \mathrm{GHz}, \quad A=0.4000$

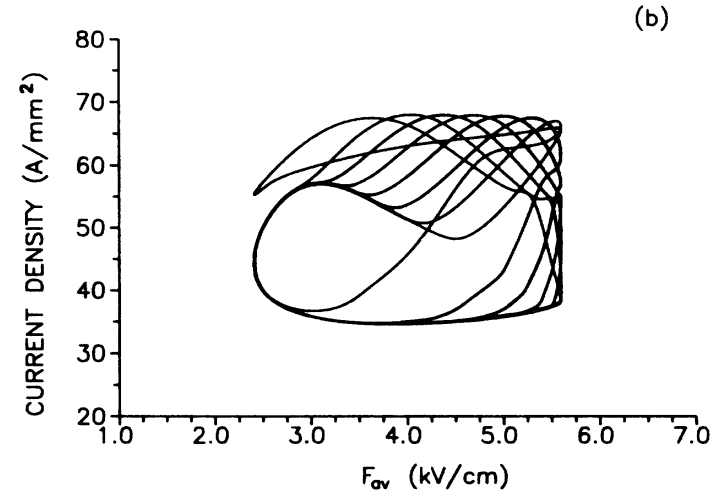

FIG. 7. Stationary 8:7 frequency-locked solution existing for $f=10.0 \mathrm{GHz}$ and $A=0.40$. (a) Temporal variation of current density, applied field, and internal field distribution; (b) corresponding phase-space trajectory.

mapped into $b_{1}, a_{2}$ into $b_{2}$, and $a_{3}$ into $b_{3}$. The mixing, which is characteristic of chaotic systems, is revealed by the interchange of the order of the points. After an additional five periods of the external signal, $b_{1}$ is mapped into $c_{1}$, etc., and the points that initially were relatively close to each other have now been scattered over a good deal of the attractor. All of the stroboscopic maps were obtained with the phase of the microwave signal equal to $\pi$, i.e., at times when the microwave signal is zero and on its way to become negative.

As the microwave amplitude is further increased, a transition to a different set of modes takes place. If the frequency of the microwave signal is relatively low $(<30$ $\mathrm{GHz}$ ), and yet higher than the undisturbed Gunn frequency $f_{\text {Gunn }}$, a quenched domain mode can be observed. This mode is illustrated in Fig. 10 for $A=0.50$ and $f=10.0 \mathrm{GHz}$. With this mode, a domain is formed and starts to propagate during a positive swing of the microwave signal. However, before the domain reaches the anode contact, the microwave signal turns negative, and because of the amplitude of this swing, the domain is quenched. With the next positive swing of the microwave signal, a new domain is formed, and the two 
$F=30.0 \mathrm{GHz}, \quad A=0.5600$
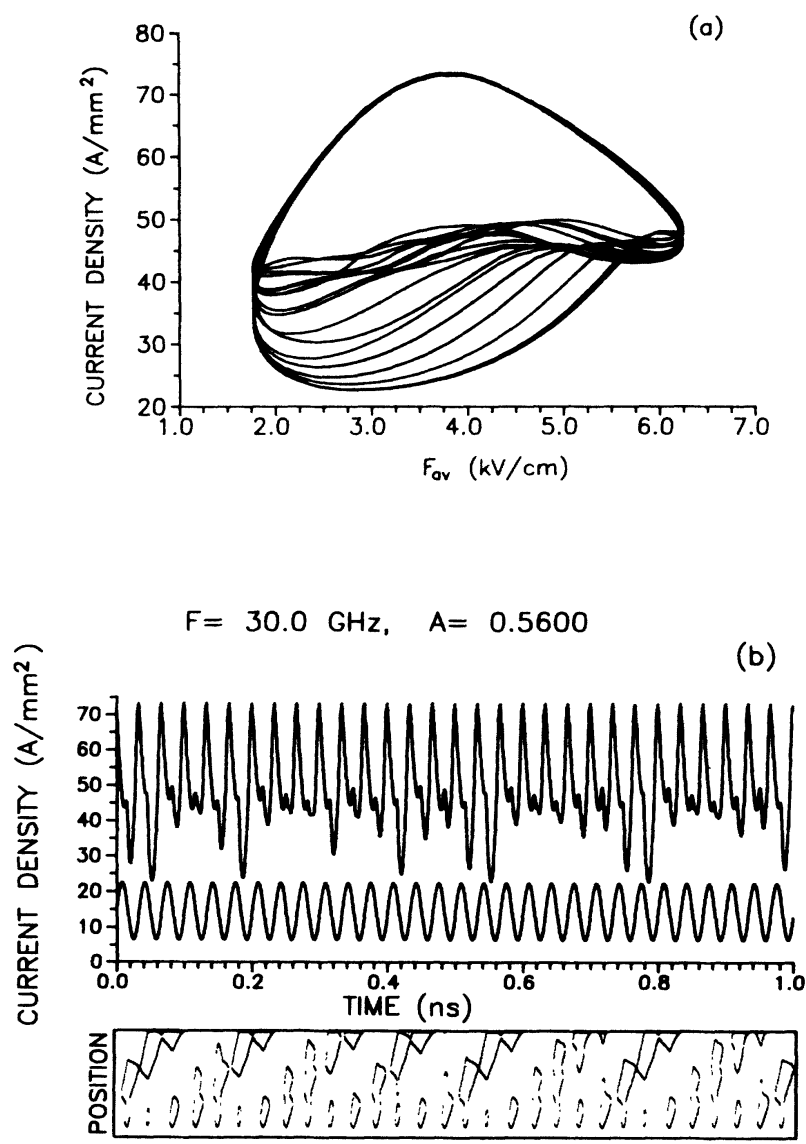

$F=30.0 \mathrm{GHz}, \quad A=0.5600$

(c)

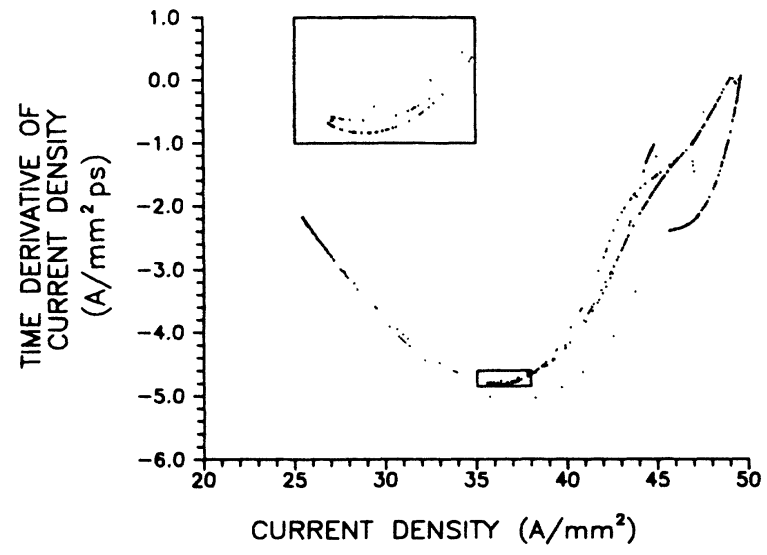

FIG. 8. (a) Phase plot of the chaotic solution existing for $f=30.0 \mathrm{GHz}$ and $A=0.56$. (b) Corresponding temporal variation of the current density and the electric-field distribution. (c) Stroboscopic map obtained by plotting simultaneous values of the current density and the rate of change of this variable each time the microwave signal has completed a full cycle.
$F=30.0 \mathrm{GHz}, \quad A=0.5600$

(a)
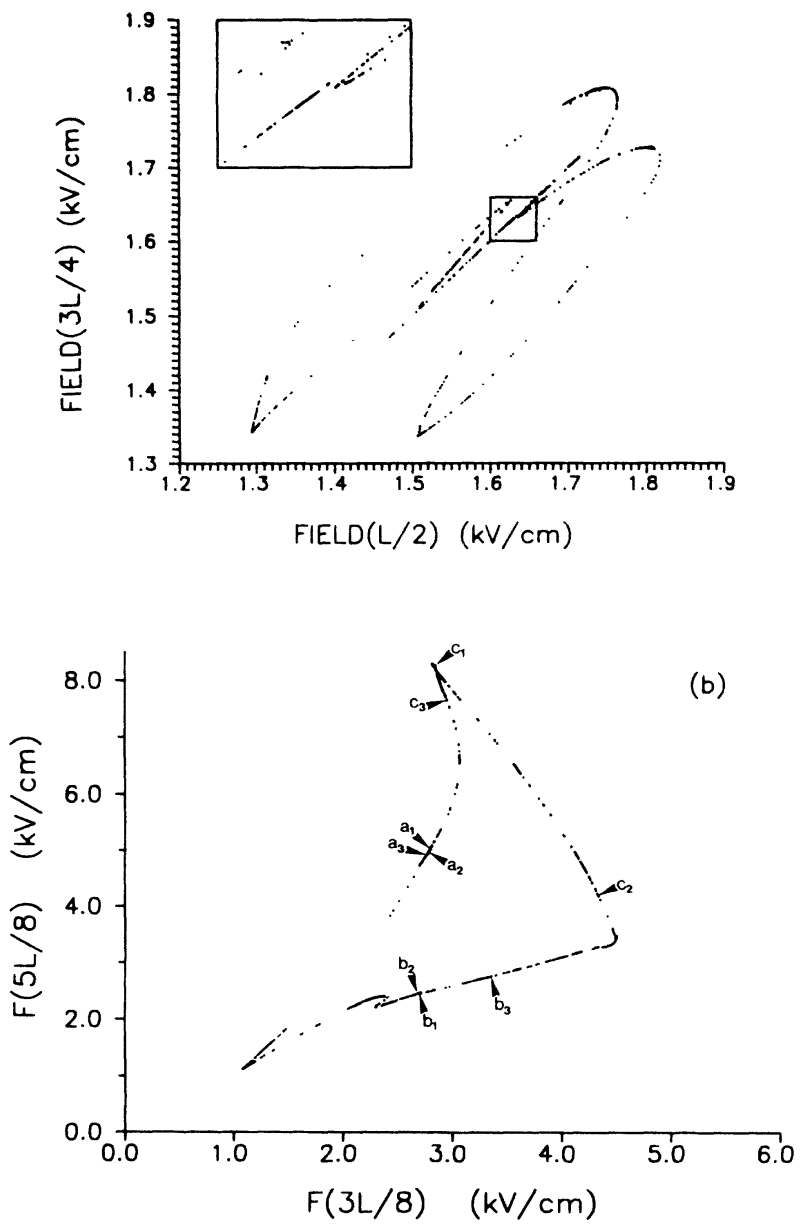

FIG. 9. (a) Stroboscopic map obtained by plotting simultaneous values of the electric field in two points of the crystal situated $x_{1}=L / 2$ and $x_{2}=3 L / 4$ from the cathode contact; (b) similar plot obtained for $x_{1}=3 L / 8$ and $x_{2}=5 L / 8$. These plots illustrate the folding and mixing properties of chaotic systems.

modes thus lock into a 1:1 solution over a relatively broad frequency interval. In the phase plot, the quenched mode is characterized by the fact that the current rises to its Ohmic value at a time where the average field is below the threshold for domain formation.

For a large amplitude microwave signal of a frequency below the undisturbed Gunn frequency, a delayed domain mode is observed. This is also a 1:1 frequencylocked solution, but now the domain is allowed to propagate all the way through the crystal. Instead, the entrainment occurs as the diode in each swing of the microwave signal awaits a sufficiently high average field before a new domain is formed.

Finally, at microwave frequencies above approximately $30 \mathrm{GHz}$, a transition to a limited space-charge accumulation (or LSA) mode takes place. This mode, which is il- 


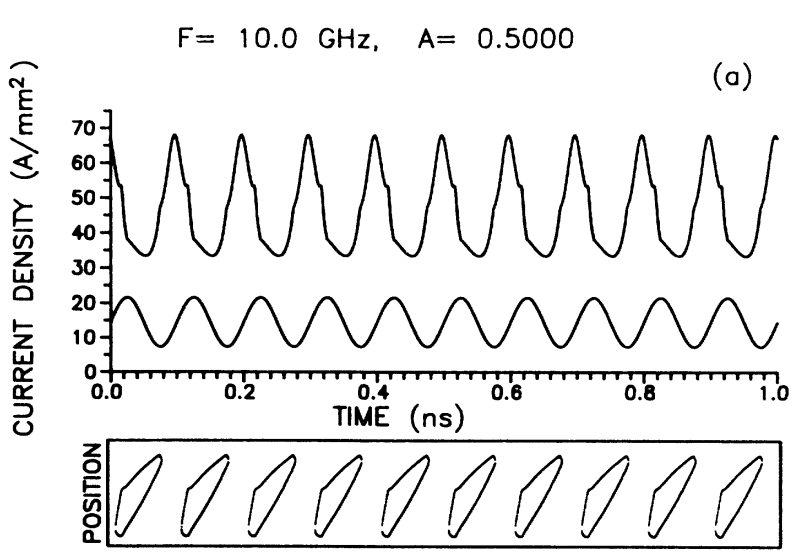

$F=10.0 \mathrm{GHz}, \quad A=0.5000$

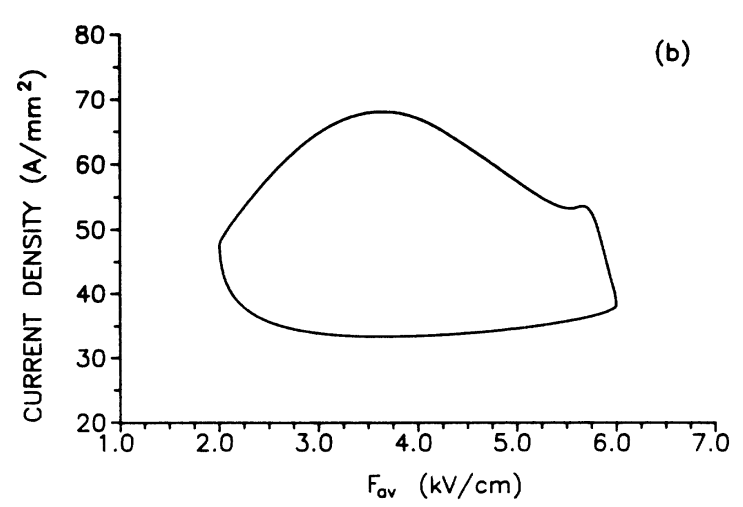

FIG. 10. (a) Quenched domain mode existing for $f=10.0$ $\mathrm{GHz}$ and $A=0.50$. Temporal variation of current density, applied field, and internal field distribution; (b) corresponding phase-space trajectory. In this mode, the domain is quenched by a negative swing of the microwave signal before it reaches the anode contact.

lustrated in Fig. 11 for $A=0.60$ and $f=55.0 \mathrm{GHz}$, is characterized by an almost complete suppression of domain formation. During a positive swing of the microwave signal, domains start to build up from irregularities or thermal noise. However, before the domains have grown sufficiently to reach macroscopic significance, the microwave signal again turns negative. The amplitude and duration of this negative swing are sufficient to allow the electron gas to relax back towards a spatially homogeneous distribution.

By performing a large number of simulations with the model, we have determined how the type of solution varies with the amplitude and frequency of the applied microwave signal. This has provided the phase diagram of Fig. 12. In this diagram, Arnol'd tongues of frequency-locked behavior are seen to arise in intervals around rational ratios of the external frequency to the internally controlled domain frequency. With increasing microwave amplitudes, the tongues broaden and start to overlap. This produces various types of mode-converting
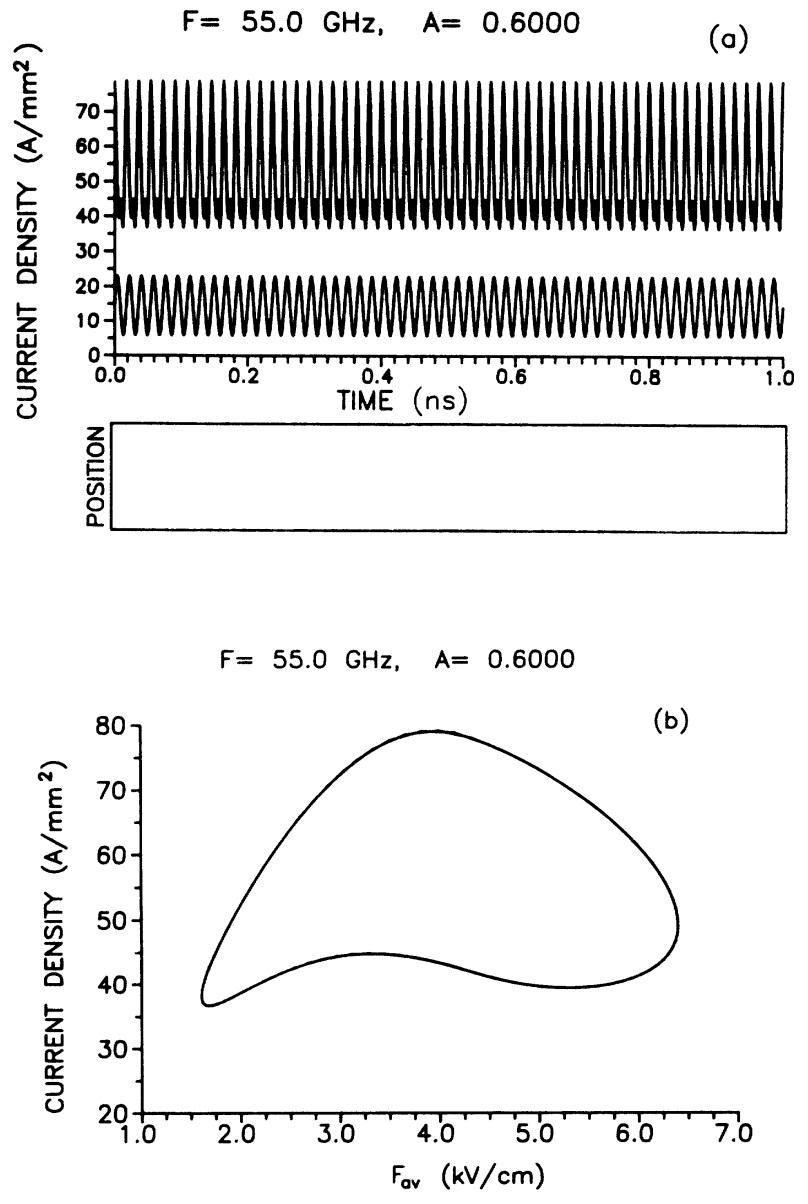

FIG. 11. Limited space-charge accumulation mode obtained for $F=55.0 \mathrm{GHz}$ and $A=0.60$. (a) Temporal variation of current density and applied field; (b) corresponding phase-space trajectory. This mode is characterized by the complete suppression of domain formation.

bifurcations and chaos. As the microwave amplitude approaches $60-70 \%$ of the applied dc field, a transition to delayed and quenched domain modes occurs for relatively low microwave frequencies, while at higher frequencies LSA oscillations are observed. Although the general conditions for obtaining some of these modes are relatively well established, the complicated distribution of behavioral forms that can arise in periodically driven Gunn diodes has not previously been studied in detail.

\section{DISCUSSION}

The developments in nonlinear science that have occurred during the past $10-15$ years have completely revolutionized our understanding of complex behavior. With recognition of the fractal geometry underlying chaotic behavior, and with the discovery of universal quantitative relations governing the onset of chaos, we can now describe the behavior of systems that were previously regarded as hopelessly complex and, for that reason, often overlooked. At the same time, a set of new 


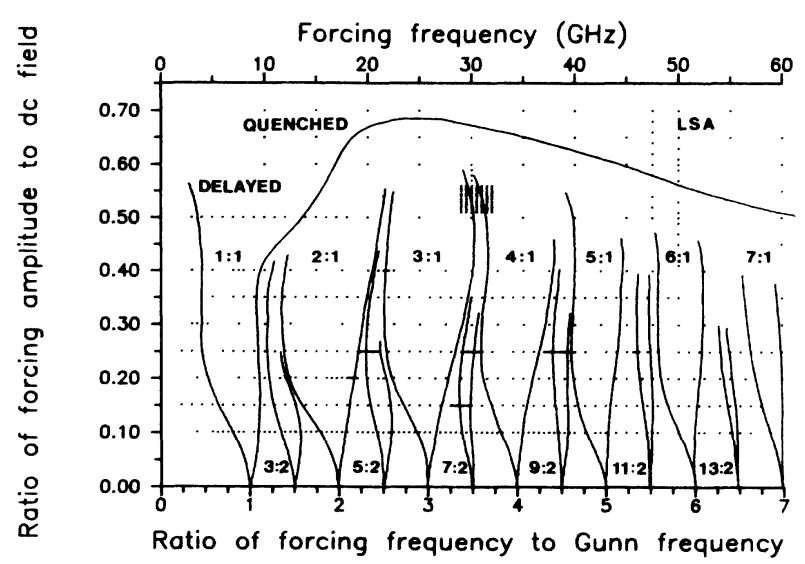

FIG. 12. Phase diagram showing the distribution of behavioral forms as a function of the frequency and amplitude of the applied microwave signal. Interspersed with the Arnol'd tongues of frequency-locked behavior, we can observe quasiperiodic behavior. At high microwave amplitudes, delayed, quenched, and limited space-charge accumulation modes are observed.

diagnostic tools for chaotic systems such as Lyapunov exponents and fractal dimensions have been developed.

Although much of the inspiration for this breakthrough came from the urge to understand turbulence, most of the results obtained so far pertain to systems governed by a few coupled ordinary differential equations or to constraint systems, where the number of modes are relatively small. However, most physical systems of interest do not belong to these classes. Usually, the spatial extension of the system means that the motion will be chaotic not only in time but also in space; i.e., that snapshots of the system will show spatial disorder.

During the past few years a number of interesting results pertaining to extended systems have started to appear. Through the study of coupled map systems, ${ }^{28-30}$ a richness of new phenomena have been discovered which relates both to low-dimensional chaos and to collective phenomena such as phase transitions.

The present study has followed a somewhat different line of approach. The aim has been to represent the physical mechanisms of the bulk negative differential resistance oscillator in sufficient detail to make the analysis applicable to real diodes. While on one hand illustrating the universal phenomenon of entrainment in nonlinear systems, the phase diagram also reflects the specifics of our problem. In particular, the transition to delayed, quenched, and limited space-charge accumulation modes are not encountered in simpler models.

\section{ACKNOWLEDGMENTS}

We would like to acknowledge Y. Ueda and M. J. Ogorzalek for their continuous interest in our work. $\mathbf{M}$. H. Jørgensen and T. Guldbrandsen are acknowledged for a number of helpful suggestions.
${ }^{1}$ Universality in Chaos, edited by P. Cvitanovic (Hilger, London, 1984).

${ }^{2}$ P. Bergé, Y. Pomeau, and C. Vidal, Order Within Chaos, Towards a Deterministic Approach to Turbulence (Wiley, New York, 1984).

${ }^{3}$ Dimensions and Entropies in Chaotic Systems, edited by G. Mayer-Kress (Springer-Verlag, Berlin, 1986).

${ }^{4}$ Chaos in Biological Systems, edited by H. Degn, A. V. Holden, and L. F. Olsen (Plenum, New York, 1987).

${ }^{5}$ Structure, Coherence and Chaos in Dynamical Systems, Proceedings of Nonlinear Science, edited by P. L. Christiansen and R. D. Parmentier (Manchester University Press, England, 1989).

${ }^{6}$ T. Matsumoto, L. O. Chua, and M. Komuro, IEEE Trans. Circuits Syst. CAS-32, 798 (1985).

${ }^{7}$ T. Matsumoto, L. O. Chua, and M. Komuro, Circuit Theory 14, 117 (1986).

${ }^{8}$ T. Saito, IEEE Trans. Circuits Syst. CAS-32, 320 (1985).

${ }^{9}$ A. S. Pikovsky and M. I. Rabinovich, Proceedings of the International Workshop on Nonlinear and Turbulent Processes in Physics, Kiev, 1979 [Physica D 2, 8 (1981)].

10J. Testa, J. Pérez, and C. Jeffries, Phys. Rev. Lett. 48, 714 (1982).

${ }^{11}$ J. B. Gunn, Solid State Commun. 1, 88 (1963).

${ }^{12}$ B. K. Ridley, Proc. Phys. Soc. London 82, 954 (1963).

${ }^{13}$ T. M. Quist and A. G. Foyt, Proc. IEEE 53, 303 (1965).
${ }^{14}$ D. E. McCumber, and A. G. Chynoweth, IEEE Trans. Electron Devices ED-13, 4 (1966).

${ }^{15}$ H. Kroemer, IEEE Trans. Electron Devices ED-13, 27 (1966).

16J. B. Gunn, IBM J. Res. Dev. 13, 591 (1969).

${ }^{17}$ J. A. Copeland, Proc. IEEE 54, 1479 (1966).

${ }^{18}$ A. R. Hutson, A. Jayaraman, A. G. Chynoweth, A. S. Coriel, and W. L. Feldman, Phys. Rev. Lett. 14, 639 (1965).

${ }^{19}$ W. Fawcett, A. D. Boardman, and S. Swain, J. Phys. Chem. Solids 31, 1963 (1970).

${ }^{20}$ P. N. Butcher, W. Fawcett, and C. Hilsum, Br. J. Appl. Phys. 17, 841 (1966).

${ }^{21}$ H. D. Rees, IBM J. Res. Dev. 13, 537 (1969).

22J. G. Ruch and G. S. Kino, Phys. Rev. 174, 921 (1968).

${ }^{23}$ R. G. Ulbrich, J. A. Kash, and J. C. Tsang, Phys. Rev. Lett. 62, 949 (1989).

${ }^{24}$ J. A. Kash, J. C. Tsang, and J. M. Hvam, Phys. Rev. Lett. 54, 2151 (1985).

${ }^{25}$ M. H. Jensen, P. Bak, and T. Bohr, Phys. Rev. A 30, 1960 (1984).

${ }^{26}$ M. H. Jensen, P. Bak, and T. Bohr, Phys. Rev. Lett. 50, 1637 (1983).

${ }^{27}$ P. Bak and J. von Boehm, Phys. Rev. B 21, 5297 (1980).

${ }^{28}$ K. Kaneko, Prog. Theor. Phys. 74, 1033 (1985).

${ }^{29}$ K. Kaneko, Physica D 23, 436 (1986).

${ }^{30}$ K. Kaneko, Physica D 34, 1 (1989). 